\title{
CARACTERIZACIÓN DE LOS SISTEMAS OPERACIONALES, MODALIDADES Y ARTES DE PESCA \\ UTILIZADOS PARA LA CAPTURA DE LA LANGOSTA \\ PANULIRUS GRACILIS (STREETS, 1871) EN GUANACASTE, COSTA RICA
}

\section{Helven Naranjo ${ }^{1}$}

\begin{abstract}
RESUMEN
Se describen las dos modalidades de pesca que se utilizan en la captura de la langosta del Pacífico Panulirus gracilis (Streets, 1871) en la región de Santa Cruz, Guanacaste, Costa Rica. Estas modalidades son el buceo a pulmón y el buceo con compresor, asociadas a un único arte de pesca llamado "bichero", arte que tiene la singularidad de ser muy versátil y permite la captura de otras especies de interés comercial. Su uso, asociado a la modalidad de pesca del buceo con compresor, podría estar ocasionando una dispersión de la langosta y una alteración en sus parámetros de crecimiento; además, deja la selectividad en las tallas que se capturan a decisión del pescador. Asimismo, se describen los equipos que se utilizan en ambas modalidades de pesca, así como la forma en que operan, incluyendo los riesgos que representa el buceo con compresor para la integridad física de los pescadores. A partir del análisis realizado, se recomiendan otros tipos de artes de pesca selectivos, considerando que los sistemas tradicionales de pesca han impactado negativamente la disponibilidad de la langosta y demás especies a través de los años.
\end{abstract}

Palabras claves: Panulirus gracilis, Costa Rica, "bichero", compresor.

\begin{abstract}
We describe two fishing methods used in the fishery for the Pacific spiny lobster Panulirus gracilis (Streets, 1871) in Santa Cruz, Guanacaste, Costa Rica, which include lung diving and "hookah" diving (using a compressor) associated with a single gear called "bichero". This fishing gear is unique in being extremely versatile as it allows capturing other species of commercial interest. However, the use of this gear under water with the "hookah" fishing modality could potentially cause lobster dispersal and affect lobster growth, and also leaves the size selectivity as a decision that has to be made by fishermen. It also describes the equipment used in both fishing styles, as well as how they operate and the physical risks posed by "hookah" for fishermen. Furthermore, we recommended other types of selective fishing gear, whereas traditional fishing systems have negatively impacted the availability of lobster and other species through the years.
\end{abstract}

Keywords: Panulirus gracilis, Costa Rica, "bichero", "hookah".

\section{INTRODUCCIÓN}

En Costa Rica, la captura de recursos pesqueros compuesta por especies bentodemersales como la langosta ha tenido un incremento considerable, debido a su demanda en el mercado local e internacional.
La langosta verde del Pacífico Panulirus gracilis es uno de los recursos mejor pagados, por lo que genera altos ingresos económicos a los pescadores. Además, su captura está asociada a la de otras especies complementarias que ayudan a maximizar la renta de los pescadores locales (Naranjo,

1 Universidad Nacional, Costa Rica. helvenn@hotmail.com

Recibido 16-II-2010

Aceptado 29-VI-2010

DOI: http://dx.doi.org/10.15359/revmar.2.6 
2009). En la región de Santa Cruz, estas pesquerías multiespecíficas utilizan artes y modalidades de captura que han sido poco documentadas, por lo cual el presente estudio pretende generar información general que permita su análisis y así contribuir a la ordenación de la actividad.

Las medidas administrativas que se aplican en la regulación de la pesquería de la langosta son: prohibir la captura de hembras ovígeras y una talla mínima de captura legal de $80 \mathrm{~mm}$ de longitud cefalotoráxica (LC). Sin embargo, Naranjo (2009) reportó en esta área un $74.9 \%$ de especímenes en la captura comercial por debajo de la talla mínima de captura y un $67.2 \%$ del total de hembras con atributos de madurez sexual. Esto quiere decir que en esta región, la pesca de la langosta está basada en la captura de juveniles sublegales y de hembras reproductivas. Esta problemática podría estar asociada al tipo de arte de pesca, ya que según Sparre et al. (1998), la talla de primera captura depende de las características del arte de pesca que se utiliza.

Por otra parte, las principales comunidades pesqueras que se dedican a la extracción de invertebrados marinos comprenden los puertos de San Juanillo, Pargos, Brasilito y Playa Lagarto, siendo este último el que aporta el mayor número de embarcaciones dedicadas exclusivamente a esta actividad. Además, muchos de los pescadores de estas comunidades costeras realizan, de manera esporádica, actividades extractivas en áreas someras asociadas al buceo a pulmón, principalmente cuando las condiciones de visibilidad en la columna de agua son favorables.

La pesca artesanal, en algunas comunidades de esta región, está siendo impactada por una fuerte presión comercial proveniente de grandes complejos hoteleros y restaurantes, que solicitan productos frescos para satisfacer las demandas gastronómicas de los turistas. Adicionalmente, el consumo local y el suministro al mercado de exportación hacen que aumenten las faenas de pesca y se invierta en esfuerzo pesquero (más pangas, equipo, motores, etc.). Esto genera problemas de sobrecapacidad, con la subsiguiente sobreexplotación de las poblaciones de valor comercial. Sobre esta base, se hace necesario evaluar cualitativamente las propiedades de las modalidades y artes utilizadas para la captura de la langosta en términos de selectividad, eficacia y forma de uso, con el fin de valorar las posibles consecuencias de estas sobre este recurso y el pescador.

\section{MATERIALES Y MÉTODOS}

Se recopiló información de campo mediante la aplicación de encuestas y entrevistas a 26 pescadores, desde el mes de noviembre del 2007 hasta octubre del 2008, en los puertos pesqueros de Playa Lagarto, San Juanillo y Pargos, con el propósito de caracterizar el tipo de equipo y las embarcaciones que se usan para la extracción de la langosta y especies complementarias. Además, durante las faenas de pesca, se realizó una caracterización de los aspectos operacionales, con el objetivo de registrar las distintas actividades que se efectúan a bordo de las embarcaciones.

También, se acompañó en alrededor de 63 inmersiones a los buzos que practican la pesca, tanto a pulmón como con compresor, con el fin de determinar la forma en que utilizan el arte de pesca con el cual realizan la captura. 
Cuadro 1. Equipo utilizado en las dos modalidades de pesca de la langosta y demás especies bentodemersales, en la región de Santa Cruz

Table 1. Equipment used in both fishing styles to catch lobster and other bentodemersal species in the region of Santa Cruz

\begin{tabular}{|c|c|c|}
\hline \multirow[b]{2}{*}{ Equipo } & \multicolumn{2}{|c|}{ Modalidades de pesca } \\
\hline & Buceo a pulmón & Buceo con compresor \\
\hline Snorkel & $\mathrm{x}$ & $\mathrm{x}$ \\
\hline Patas de rana & $\mathrm{x}$ & $\mathrm{x}$ \\
\hline Máscara & $\mathrm{x}$ & $\mathrm{x}$ \\
\hline Malla & $\mathrm{x}$ & $\mathrm{x}$ \\
\hline Guantes & $\mathrm{x}$ & $\mathrm{x}$ \\
\hline Arbaleta* & $\mathrm{x}$ & $\mathrm{x}$ \\
\hline Cuchillo & $\mathrm{x}$ & $\mathrm{x}$ \\
\hline Bichero & $\mathrm{x}$ & $\mathrm{x}$ \\
\hline Martillo & & $\mathrm{x}$ \\
\hline Manguera & & $\mathrm{x}$ \\
\hline Compresor & & $\mathrm{x}$ \\
\hline Regulador & & $\mathrm{x}$ \\
\hline Pesas & & $\mathrm{x}$ \\
\hline Cinturón de buceo & & $\mathrm{x}$ \\
\hline
\end{tabular}

* Equipo opcional.

\section{RESULTADOS}

\section{Caracterización de los métodos y arte de pesca}

Tradicionalmente, las modalidades de pesca utilizadas para la captura de la langosta en la región de Santa Cruz: son el buceo libre o a pulmón y el buceo con compresor. En el primero se utiliza solamente equipo básico: aletas o patas de rana, snorkel, máscara o visor de buceo, malla, guantes, arbaleta, cuchillo y gancho o "bichero". El tiempo efectivo de la pesca es muy variable y puede oscilar entre 2 y 5 horas. Usualmente se opera a profundidades no mayores a los $11 \mathrm{~m}$, dependiendo de la capacidad y habilidad del pescador.

El equipo utilizado para el buceo con compresor comprende: aletas o patas de rana, cinturón de buceo, máscara o visor de buceo, malla, guantes, arbaleta, martillo o mazo, pesas, cuchillo y gancho o "bichero" (Cuadro 1). El tiempo efectivo de la pesca puede durar entre 3 y 5 horas, con etapas de descanso de 10 a 30 minutos entre inmersiones. Generalmente trabajan dos buzos a un mismo tiempo y raras veces trabajan solos o tres buzos por compresor en una misma inmersión.

El compresor que suministra el aire al buzo está constituido básicamente por tres componentes: el tanque, el motor y el compresor (Fig. 1). El motor utilizado, en la mayoría de compresores en esta región, es de una potencia de 5 HP y todos son adaptados para que funcionen con gasolina. La capacidad del tanque del compresor es de aproximadamente 250 libras por pulgada cuadrada. El aceite que se utiliza en el motor del compresor en general es mineral.

El compresor se conecta a una boquilla de buceo por un manguera de $1 / 4$ de pulgada de 


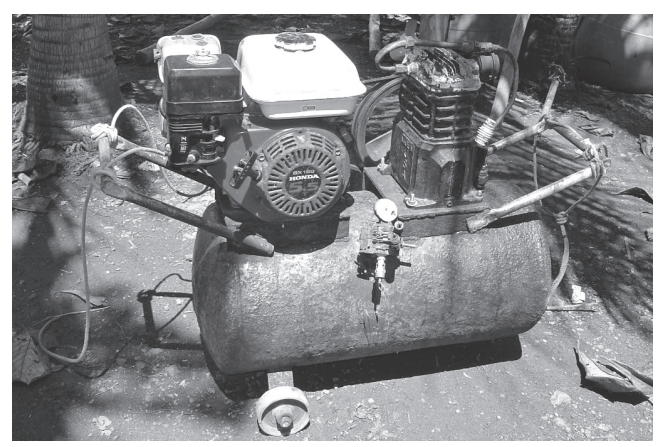

Fig. 1. Compresor utilizado como método de pesca en la captura de la langosta $P$. gracilis y demás recursos bentodemersales en la región de Santa Cruz, Guanacaste

Fig. 1. Compressor used in the "hooka" fishing style to catch lobster and other bento-demersal species in the region of Santa Cruz

diámetro. Dicha manguera es la que lleva el aire al buzo y consta de dos secciones, la primera que alcanza una longitud aproximada de $100 \mathrm{~m}$ y se divide por medio de un dispositivo en forma de T. La segunda parte es de dos o tres secciones que miden 50 m cada una y les permite a los buzos libertad para moverse de forma individual bajo el agua.

El arte de pesca utilizado por ambas modalidades es el gancho o "bichero" (Fig. 2). Esta herramienta está formada por una varilla de acero inoxidable o hierro, de 0.6 $\mathrm{cm}$ de ancho y de aproximadamente 1.20 $\mathrm{m}$ de largo, con una curva pronunciada en forma de gancho en un extremo. Algunos utilizan un anzuelo sujeto con un cable o varillas de mayor longitud, que pueden medir hasta $2.5 \mathrm{~m}$, las cuales permiten capturar organismos que se esconden en cavernas profundas. A esta varilla se le enrolla una banda de caucho o neumático, que cumple la función de mango, que es por donde se sostiene el arte.

El gancho o "bichero" es de uso generalizado por los buzos artesanales de esta zona y tiene la singularidad de ser muy versátil, ya que con él también se pueden capturar otras especies, como el pulpo (Octopus vulgaris), ostión (Spondylus calcifer y $S$. princeps), y peces de las familias lutjanidae, serraidae, balistidae y scorpenidae. La eficiencia del "bichero" está condicionada a la habilidad que tenga el pescador en el manejo de este $\mathrm{y}$, además, la selectividad en las tallas que se capturan queda sujeta también al pescador y no a este tipo de arte de pesca no selectivo.

\section{Caracterización del sistema operacional del buceo con compresor y buceo a pulmón}

Las faenas de pesca de langosta utilizando la modalidad del buceo con compresor son efectuadas por tres tripulantes (dos buzos y un botero) y la duración por viaje de pesca comprende entre 5 y 7 horas. El botero es el que se encarga de dirigir el motor de la embarcación durante las faenas de buceo, además de vigilar la condición de las mangueras que llevan el aire a los buzos durante las inmersiones y el procesamiento de la captura.

Fig. 2. Arte de pesca ("bichero") utilizado para la captura de la langosta P. gracilis y demás recursos bentodemersales

Fig. 2. Fishing art used to catch lobster and other bento-demersal species in the region of Santa Cruz 

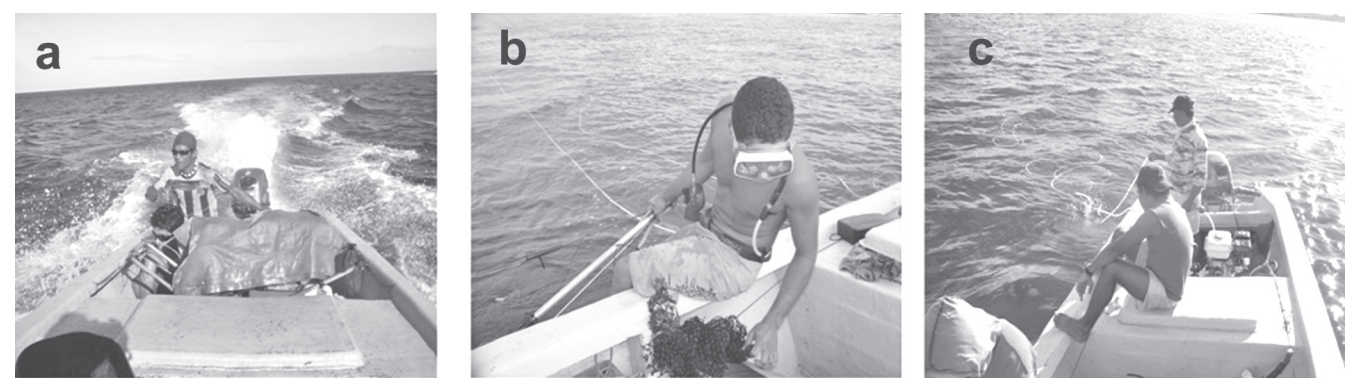

Fig. 3. Localización del caladero (a). Preparación del equipo de buceo (b). Despliegue de las mangueras (c)

Fig. 3. Location fishing ground (a). Preparing diving equipment (b). Deployment hoses (c).

Las operaciones de pesca siguen un orden, que se puede esquematizar en varias etapas sucesivas. Estas etapas corresponden a: la elección y localización del área de pesca o caladero, la preparación del compresor y equipo, el número de inmersiones $\mathrm{y}$, por último, el procesamiento de la captura.

En la primera etapa se selecciona el área de buceo; algunos pescadores localizan los caladeros orientándose al navegar mediante triangulación, utilizando marcas en la tierra; otros buscan los lechos rocosos arrastrando el ancla sobre el fondo. Generalmente, en un mismo viaje se bucea en lugares diferentes, dependiendo del número de inmersiones. Una vez seleccionado el sitio, se despliega la manguera que lleva el aire a los buzos, se prepara el compresor y los buzos alistan su equipo (Fig. 3).

Se realizan entre 1 y 4 inmersiones por día, dependiendo de la profundidad, el sitio de buceo, la visibilidad, las corrientes, el estado anímico del buzo y los rendimientos parciales. Mientras los buzos se encuentran operando, el botero los sigue, guiándose por las burbujas que emergen a la superficie y procurando que la manguera no se enrede. Los buzos van depositando la captura en sus respectivas mallas y esta suele ser muy diversa, desde peces de escama hasta pepinos de mar. Al momento de emerger, el botero ayuda a los buzos a embarcar la captura para luego vaciarla en un recipiente con agua de mar, destinado para ese fin (Fig. 4).

Una vez terminadas las inmersiones, o bien, durante los lapsos de estas, se efectúa el procesamiento de la captura a bordo de la embarcación por parte del botero y/o los buzos. Este consiste en el eviscerado, limpieza y desconche de las distintas especies, que luego son almacenadas en el recipiente con agua de mar sin hielo, la cual se cambia regularmente hasta llegar a puerto.
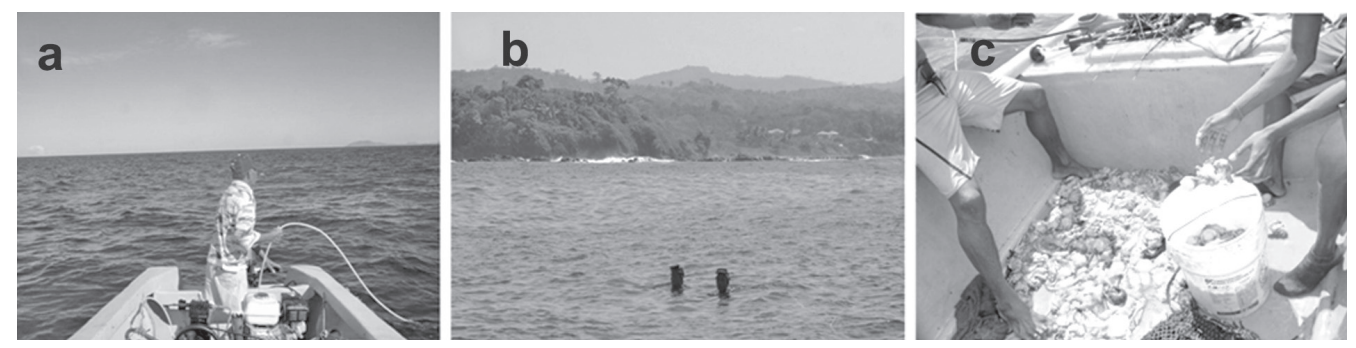

Fig. 4. Botero dirigiendo la embarcación y la manguera (a). Buzos emergiendo con la captura (b). Procesamiento de la captura (c)

Fig. 4. Fisherman guiding the boat (a). Divers emerge with the capture (b). Processing captures (c) 
Usualmente no se realizan inmersiones a más de $30 \mathrm{~m}$ de profundidad, no obstante, se observó que a medida que la productividad de los caladeros disminuye, los buzos tienden a trabajar en áreas más profundas y también a aumentar sus horas bajo el agua en su búsqueda de la captura. Esto trae como resultado un mayor riesgo para los pescadores de sufrir problemas de descompresión, así como un aumento en los costos operacionales por embarcación.

A diferencia del buceo profesional, militar, científico o recreativo, en el que se utilizan tablas o computadoras para limitar el tiempo bajo el mar en función de la profundidad de la inmersión, los pescadores locales carecen de este tipo de medios complejos de orientación. En cambio, estiman la profundidad basándose en la longitud de la cuerda que sujeta al ancla o en su conocimiento del área. En lugar de controlar el tiempo, la mayoría de los buceadores permanecen bajo la superficie hasta haber obtenido un considerable volumen de captura en sus mallas.

Las embarcaciones que se utilizan en esta región, asociadas al uso del compresor, son conocidas como pangas y presentan una eslora reducida, sin cubierta; están construidas de madera, fibra de vidrio, resina y playwood. Poseen una capacidad máxima de tres tripulantes y una autonomía también reducida.

Estas embarcaciones son impulsadas con motores fuera de borda de 25 a $45 \mathrm{HP}$, la mayoría de cuatro tiempos. Aun cuando la potencia de estos motores permitiera realizar travesías relativamente largas, dichas embarcaciones no poseen equipos de navegación ni están adaptadas para viajes de varios días en el mar.

Por otra parte, los buzos a pulmón trabajan en áreas someras, cercanas a la costa. Usualmente operan en parejas o en tríos y rara vez bucean solos. Las áreas de buceo son seleccionadas según el nivel de la marea, el oleaje, la visibilidad, el conocimiento que tengan los buzos del área y la lejanía de la zona al centro de acopio, entre otros factores. La duración del viaje de pesca puede oscilar entre 1 y 4 horas y los principales recursos que se extraen en estas áreas, además de la langosta, son el pulpo y los peces como el loro (Scarus perico y $S$. ghobban).

\section{DISCUSIÓN}

De acuerdo con la información recopilada en los principales puertos pesqueros de la región de Santa Cruz, se comprobó que la extracción de la langosta $P$. gracilis se realiza mediante dos tipos de modalidades de pesca, el buceo con compresor y el buceo a pulmón, y un único arte de pesca, el "bichero".

Según testimonios de los pescadores de mayor edad, el buceo a pulmón es una modalidad de pesca más antigua, practicada desde la década de 1960. Su uso en las áreas de poca profundidad ha sido parte importante en las actividades extractivas de estas comunidades costeras, cuyas capturas no solo se han usado con fines comerciales sino que, principalmente, para proporcionar alimento a muchas familias. Un estudio realizado en esta región (Naranjo, 2009) comprobó que esta forma de extracción conlleva bajos costos operacionales, lo que la hace más accesible a muchos pobladores autóctonos dedicados a estas actividades de pesca de subsistencia.

Por otro lado, el uso del compresor, como modalidad de pesca, se empezó a utilizar desde hace más de veinte años, lo que permitió la expansión de las actividades de buceo hacia zonas más profundas y sobre hábitats menos explotados. Debido a 
la capacidad que tiene este mecanismo de proporcionarles un flujo de aire continuo a los buzos, se aumentaron las horas de inmersión y, por ende, los tiempos efectivos de pesca. Lo anterior resultó en un mejoramiento en la eficiencia, que se vio reflejado en los rendimientos de las capturas.

Los efectos del incremento en la eficiencia de pesca conllevan, a mediano y largo plazo, a cambios en la abundancia de los recursos (Brown et al. 1994). Sin embargo, la escasa información histórica de capturas, que existe para esta zona, hace que se desconozca el posible impacto sobre los recursos que pudo causar esta innovación tecnológica a través de dichos años.

El buceo con compresor en esta zona es una práctica que conlleva muchos riesgos, debido a que los pescadores la realizan de forma empírica y algunas veces les ocasiona graves lesiones laborales con importantes secuelas de discapacidad, daños irreparables y hasta la muerte, sin que tengan el mínimo respaldo y protección patronal del Estado o de instituciones afines. Además, la poca regulación que existe sobre esta actividad hace que los riesgos asociados al buceo con compresor sean aún mayores.

Los problemas más comunes que provoca el desconocimiento de técnicas de inmersión, cuando se bucea con compresor, son los peligros inherentes a la enfermedad del buzo o de la descompresión. También ocurren cuando falla el compresor o la manguera se enreda, lo que corta súbitamente el suministro de aire al buzo, obligándolo a nadar hacia la superficie rápidamente.

Gold (2008) realizó una encuesta en Tailandia sobre buzos que trabajan en similares condiciones que los pescadores de esta zona, donde encontró que un $85 \%$ de estos sufrían de dolores como consecuencia de su actividad, una altísima tasa de mortalidad y un gran porcentaje de buceadores activos e inactivos discapacitados a causa de las inmersiones. En la región de Santa Cruz se han reportado varios accidentes de buceo que comprenden: barotraumas, embolismo, intoxicación por monóxido de carbono, descompresión e incluso ataques de fauna marina a buzos.

Además, se desconoce la calidad del aire proporcionado por los compresores que se utilizan en esta región y si estos son aptos o no para proporcionar respiración artificial. El sistema de filtros y el tanque no están certificados ni diseñados para tal fin, por lo que es necesario que se evalúe técnicamente su uso, según las normas internacionales existentes para tales fines.

\section{Arte de pesca}

El arte de pesca utilizado en esta región es el "bichero", el cual es una herramienta versátil, que permite capturar organismos que se encuentran dentro de cavernas y adheridos a las rocas. Usualmente, las langostas son enganchadas por la hendidura que separa el abdomen del cefalotórax y arrastradas lentamente desde sus escondites, ejerciendo una considerable presión, hasta un lugar abierto cuando el buzo pueda tomarlas con la mano. Es precisamente en este último momento, y no antes, cuando el pescador tiene la posibilidad de observar, con mayor precisión, el tamaño del espécimen y si tiene o no masa ovígera. Sin embargo, en muchas ocasiones, las langostas se encuentran en lugares donde resulta difícil su manipulación u observación, como por ejemplo: cavernas angostas y profundas, sustratos con fondo de grava y arena de poca visibilidad, cobertura algal espesa, corrientes y oleajes fuertes. Esta situación hace que algunas veces sea muy difícil para el pescador no dañar las langostas antes de hacer 
una correcta discriminación de su tamaño y estado reproductivo, por lo cual se opta por llevar a la malla todo aquel organismo que fuese eventualmente lesionado.

Esta misma situación se presenta con otras especies objetivo de pesca, que también son de hábitos crípticos (como el pulpo) y resulta difícil para el pescador identificar su talla y evitar alguna lesión en el proceso de captura. De esta manera, resulta claro que el "bichero" no ayuda al pescador a realizar una correcta selección de las tallas, sin embargo, debido a su versatilidad, permite capturar otras especies de interés comercial y según la forma en que se opere, resulta ser muy eficaz. Posiblemente, estas dos últimas características permitieron que el uso de esta herramienta se generalizara con rapidez a lo largo de las zonas costeras de esta región, donde no se reporta el uso de artes alternativas como nasas, trampas o "esling".

El "esling" es una varilla liviana y fuerte, de aproximadamente un metro de longitud, con una soga de alambre en forma de anillo en un extremo. Para la captura de la langosta, los buceadores deben deslizar la soga sobre su cola, engancharla con fuerza y sostenerla con la mano libre hasta la superficie. Este arte tiene la ventaja de mantener el recurso vivo y evita las lesiones importantes a los organismos.

Según Cochrane (2005), las herramientas de captura como el "bichero" se clasifican como artes de pesca activas, con las cuales se da una persecución activa de la especie objetivo de pesca. Son análogas a los arpones y lanzas que provocan pocos daños en el ecosistema, permiten una alta selección de especies y tallas y presentan una mortalidad incidental moderada. Sin embargo, como pudimos constatar en esta investigación, dicho arte de pesca dificulta controlar la composición de tallas de captura, por lo que se comprueba su carácter de no selectivo.

Además, en otras pesquerías donde se captura la langosta utilizando instrumentos similares al "bichero" y los arpones, tiende a existir una dispersión importante de la población de langostas desde áreas someras, aumentando, de esta manera, la mortalidad natural al tener estas que desplazarse mayores distancias en su búsqueda de pareja, situación que incrementa los riesgos de ser depredadas (Davis, 1997). Esto podría provocar, a mediano plazo, un descenso de captura por unidad de esfuerzo, lo que perjudicaría tanto al recurso como al pescador, que vería mermadas sus ganancias.

También, hay que tener en cuenta el impacto potencial del "bichero" sobre las langostas que logran escapar. Durante los desembarques y en los muestreos a bordo de las embarcaciones, se comprobó la presencia de ciertos ejemplares con lesiones o con ausencia de algún apéndice. Se ha comprobado que este tipo de manipulación por pesca retarda el crecimiento de un $15 \%$ a un $50 \%$ (Waugh, 1981). En algunos casos, langostas con lesiones tienen intervalos más reducidos y tasas de crecimiento menores, comparadas con langostas sin lesiones (Briones-Fourzán y Lozano, 2003). De esta manera, se necesitan estudios que permitan cuantificar la magnitud de este fenómeno y su grado de afectación sobre los parámetros de crecimiento.

\section{Recomendaciones}

Se recomienda el uso de las nasas o trampas, ya que son artes de pesca que permiten controlar la composición de tallas de captura, por medio del tamaño de la luz de la malla o el ancho del tipo de enrejado, lo que daría la oportunidad de 
liberar hembras ovígeras y, además, se podrían utilizar para pescar en zonas más profundas y disminuirían el riesgo para los pescadores al sustituir las faenas del buceo y los costos asociados a esta modalidad de pesca. Según Ramírez (1996), la utilización de trampas conlleva una disminución de la captura de langostas de talla sublegal y tiende a mejorar la tasa de captura de langostas legales, por medio de la selección mecánica de las tallas, que está en función del tamaño de la malla, de las dimensiones de la entrada y de la presencia de ventanas para escape (Krouse, 1989).

Sin embargo, las desventajas que tendrían las nasas o trampas, con respecto a la captura con equipo de buceo y el "bichero", son que tienden a ser artes que capturan pocas especies a la vez, lo que sería poco rentable con el actual estado de explotación del recurso y dejaría por fuera otras especies que representan ingresos importantes a los pescadores. Además, implicaría una inversión económica inicial más alta. Estos impactos económicos habría que tenerlos en cuenta a la hora de implementar artes de pesca alternativas.

Sería recomendable que las autoridades encargadas de la administración de los recursos pesqueros en esta región, promovieran proyectos encaminados a la búsqueda e implementación de artes de pesca selectivas como las nasas, el "esling", y refugios artificiales para langosta, con el fin de controlar las tallas en las capturas, la reducción de lesiones a las langostas durante el proceso de captura causadas por el "bichero", la disminución de los riesgos laborales asociados al buceo y reducir la mortalidad natural de organismos que transitan sobre fondos arenosos.

Con estas recomendaciones se pretende sugerir otras artes y modalidades de pesca más adecuadas y responsables, que sustituyan el buceo y, además, que permitan la sostenibilidad del recurso a largo plazo, tomando en consideración que los sistemas tradicionales de pesca posiblemente han impactado de forma negativa la disponibilidad de la langosta y demás especies a través de los años.

\section{CONCLUSIONES}

En la región de Santa Cruz, la extracción de la langosta $P$. gracilis y otras especies bentodemersales se realiza por medio de dos tipos de modalidades de pesca: el buceo con compresor y el buceo a pulmón.

El arte de pesca que se utiliza para la captura de P. gracilis es el "bichero", el cual es una herramienta que ofrece una gran versatilidad, eficacia y sirve para capturar otras especies de interés comercial. Sin embargo, deja la selectividad de las tallas que se capturan a decisión del pescador y podría estar afectando los parámetros de crecimiento de la langosta.

Las dos modalidades de extracción de la langosta $P$. gracilis en las zonas costeras de la región de Santa Cruz se realizan en estratos de profundidad, donde el buceo a pulmón se encuentra asociado a zonas someras, cercanas a la costa, y el buceo con compresor está asociado también a áreas someras y, simultáneamente, a áreas arrecifales profundas.

El buceo con compresor es una modalidad de pesca que implica riesgos a la integridad física de los pescadores, que muchas veces desconocen los correctos procedimientos de descompresión. Además, la calidad del aire generado por este sistema es dudosa y posiblemente esta modalidad de pesca está provocando, aunado al uso del arte de pesca, una dispersión importante de la población de langosta en los estratos de profundidad donde opera. 


\section{AGRADECIMIENTOS}

Extiendo un especial agradecimiento a los pescadores de Playa Lagarto, quienes hicieron posible la realización de los estudios llevados a cabo en el área: Luis Calderón, Jeudi Ortiz, Junior Espinoza, Leiter y a todos aquellos que, de una u otra forma, colaboraron en esta investigación. En la Universidad Nacional agradezco el apoyo de Luis Ángel Palacios, Rosa Soto, Luis Villalobos, Manuel Espínola, Luis Adrián Vega, Luis Sierra y Rigoberto Víquez. En el CINVESTAV a Silvia Salas, por sus oportunas aclaraciones.

\section{BIBLIOGRAFÍA}

Briones-Fourzán, P. \& Lozano, E. (2003). Factors affecting growth of the spiny lobster Panulirus gracilis and Panulirus inflatus (Decapoda: Palinuridae) in Guerrero, México. Rev. Biol. Trop., 51 (1), 165-174.

Brown, R., Caputi, N. \& Hall, N. (1994). Measurement of catch and fishing effort in the western rock lobster fishery. En B. F. Phillips, J. S. Cobb \& J. Kittaka (Eds.), Spiny Lobster Management (pp. 262-283). England: Blackwell Scientifics Publications.

Cochrane, K. L. (2005). Guía del administrador pesquero. Medidas de ordena- ción y su aplicación. FAO Documento Técnico de Pesca. No. 424. Roma: FAO. Davis, G. E. (1997). Effects of recreational harvest o a spiny lobster Panulirus argus population. Bull. Mar. Sci., 27, 233-236.

Gold, D. (2008). Cuando el dolor formaba parte del trabajo. Trabajo Revista OIT, 36, 26-27.

Krouse, J. S. (1989). Performance and selectivity of trap fisheries for crustaceans. En J. F. Caddy (Ed.), Marine Invertebrate Fisheries: Their Assessment and Management (pp. 307-326). New York, USA: John Wiley and Sons.

Naranjo, M. H. (2009). Análisis de la pesquería de la langosta Panulirus gracilis (Streets, 1871) en la zona de Santa Cruz, Guanacaste, Costa Rica. Tesis de Maestría no publicada. Universidad Nacional.

Ramírez, M. R. (1996). Ventanas de escape en trampas para la captura de la langosta roja Panulirus interruptus, en Baja California Sur, México. Cien. Mar., 22, 235-243.

Sparre, P., Ursin, E. \& Venema, C. S. (1998). Introduction to tropical fish stock assessment. Part 1: Manual FAO. Documento Técnico de Pesca. Roma: FAO.

Waugh, G. H. (1981). Management of juvenile spiny lobster (Panulirus argus) based on estimated biological parameters from Grand Bahama Island Bahamas. Proc. Gulf Caribb. Fish. Inst., 33, 271-289. 